D.T. Wickramasinghe, L. Ferrario, and G.V. Bicknell, eds.

\title{
Accretion Disks with Convective Viscosity
}

\author{
J. Smak
}

Copernicus Astronomical Center, Polish Academy of Sciences Bartycka 18, 00-716 Warsaw, Poland

\begin{abstract}
.
Models of vertical structure are calculated for a range of parameters applicable to dwarf novae with two types of viscosity being included: the standard $\alpha$-disk viscosity and the additional turbulent viscosity in the convective regions. The resulting surface density $(\Sigma)$ vs. effective temperature $\left(T_{e}\right)$ relations, compared to those without convective viscosity, show larger separation between the two critical points of the $\Sigma-T_{e}$ relation, $\Sigma_{\min }$ and $\Sigma_{\max }$. This could suggest that with such a modification the dwarf nova outbursts could be reproduced with a single value of $\alpha$. (Note that in the standard $\alpha$ approach two different $\alpha$ 's are needed on the hot and cool branch of the $\Sigma-T_{e}$ relation, with $\alpha_{\text {cool }} \approx \alpha_{h_{o t}} / 4$ ). It turns out, however, that this is not the case. This is due to the fact that additional convective viscosity makes also $\Sigma_{\max }$ smaller than in the pure $\alpha$ case.
\end{abstract}

\section{Introduction}

Dwarf nova outburst are due to the thermal instability which develops in accretion disks at low temperatures as a result of the ionization of hydrogen. From a technical point of view this instability is connected with the shape of the surface density vs. effective temperature $\left(\Sigma-T_{e}\right)$ relation obtained from integrations of equations describing the vertical structure of the disk. This involves adopting a prescription for the viscosity, which - in the standard approach - is the so-called $\alpha$-disk viscosity. The resulting $\Sigma-T_{e}$ relations show a characteristic S-shape, with its mid part, between $\Sigma_{\min }$ and $\Sigma_{\max }$, being thermally unstable.

The $\Sigma-T_{e}$ relation then becomes an essential ingredient in the timedependent calculations of the disk evolution throughout the dwarf nova cycle. Early in the game it was found that - within the standard $\alpha$ approach - it is necessary to assume two different $\alpha$ 's on the hot and cool branches of the $\Sigma-T_{e}$ relation, with $\alpha_{\text {cool }} \approx \alpha_{\text {hot }} / 4$ (e.g., Osaki 1996). In technical terms such a difference in $\alpha$ implies that the separation between the two critical points in the $\Sigma-T_{e}$ relation, i.e. $\Delta \log \Sigma=\log \Sigma_{\max }-\log \Sigma_{\min }$, should be larger than in the case $\alpha_{\text {cool }}=\alpha_{\text {hot }}$. 


\section{Disks with Convective Viscosity}

Long time ago it was suggested by Paczyński (1976) that one of the natural sources of viscosity in accretion disk could be the turbulent viscosity in their convective regions. Its efficiency in transporting the angular momentum across the disk still remains an open question (e.g. Stone and Balbus 1996 and references therein). Nevertheless several models have been calculated involving such viscosity, usually as the only source of viscosity in the convective regions, with or without standard $\alpha$-disk viscosity prescription being adopted in the radiative regions (e.g. Cannizzo and Cameron 1988, Duschl 1989, Lin and Papaloizou 1980, Smak 1982).

It appears equally logical to assume that the $\alpha$-disk viscosity operates in both - radiative and convective regions, with the turbulence being an additional source of viscosity in the convective regions. The relevant question here (and the main motivation for the present work) is whether this could modify the $\Sigma-T_{e}$ relation in such a way as to make introducing two different $\alpha$ 's, i.e. $\alpha_{\text {cool }}<\alpha_{\text {hot }}$, unnecessary. In order to answer this question, models of the vertical structure of disks have been computed for a range of parameters applicable for dwarf novae, with two types of viscosity being included

- the standard $\alpha$-disk viscosity in all regions of the disk, described with a single parameter $\alpha$, and

- the additional turbulent viscosity in the convective regions.

A more detailed descriptions of these models will be given in a paper to appear in Acta Astronomica. Two points, however, should be mentioned here with reference to the treatment of the convective regions. First, that the mixing length $\ell$ in the outer parts of the disk (i.e. at large values of $z$ ) was computed using the standard mixing-length formalism. The mixing length calculated in this way increases with decreasing $z$, and - at a certain value of $z=z_{*}$ - it becomes $\ell_{*}=2 z_{*}$. Consequently, a constant value of $\ell=\ell_{*}$ was adopted in the central parts (i.e. for $z<z_{*}$ ). Secondly, the efficiency of the convective viscosity could be taken into account by introducing an efficiency parameter $\beta \leq 1$. However, in calculations reported here it was assumed that $\beta=1$.

\section{The $\Sigma-T_{e}$ Relations}

The resulting $\Sigma-T_{e}$ relations (Figure 1) show that in the case of models with additional convective viscosity the separation in $\Sigma$ between the two critical points of the $\Sigma-T_{e}$ relation, i.e. $\Delta \log \Sigma=\log \Sigma_{m a x}-\log \Sigma_{\min }$, is indeed larger than in the pure $\alpha$ case with $\alpha_{\text {cool }}=\alpha_{\text {hot }}$. Note, however, that at the same time $\Sigma_{\max }$ becomes much smaller. Qualitatively these effects can easily be understood: in the temperature range where convection occurs the effective viscosity becomes higher and this means that the amount of material (i.e. the surface density) needed to dissipate the amount of energy corresponding to a given $T_{e}$ can become smaller.

The $\Sigma-T_{e}$ relations could now be used to calculate the time-dependent disk models aiming at reproducing the dwarf nova behavior. The results, however, was negative. The situation turns out to be qualitatively the same as in 


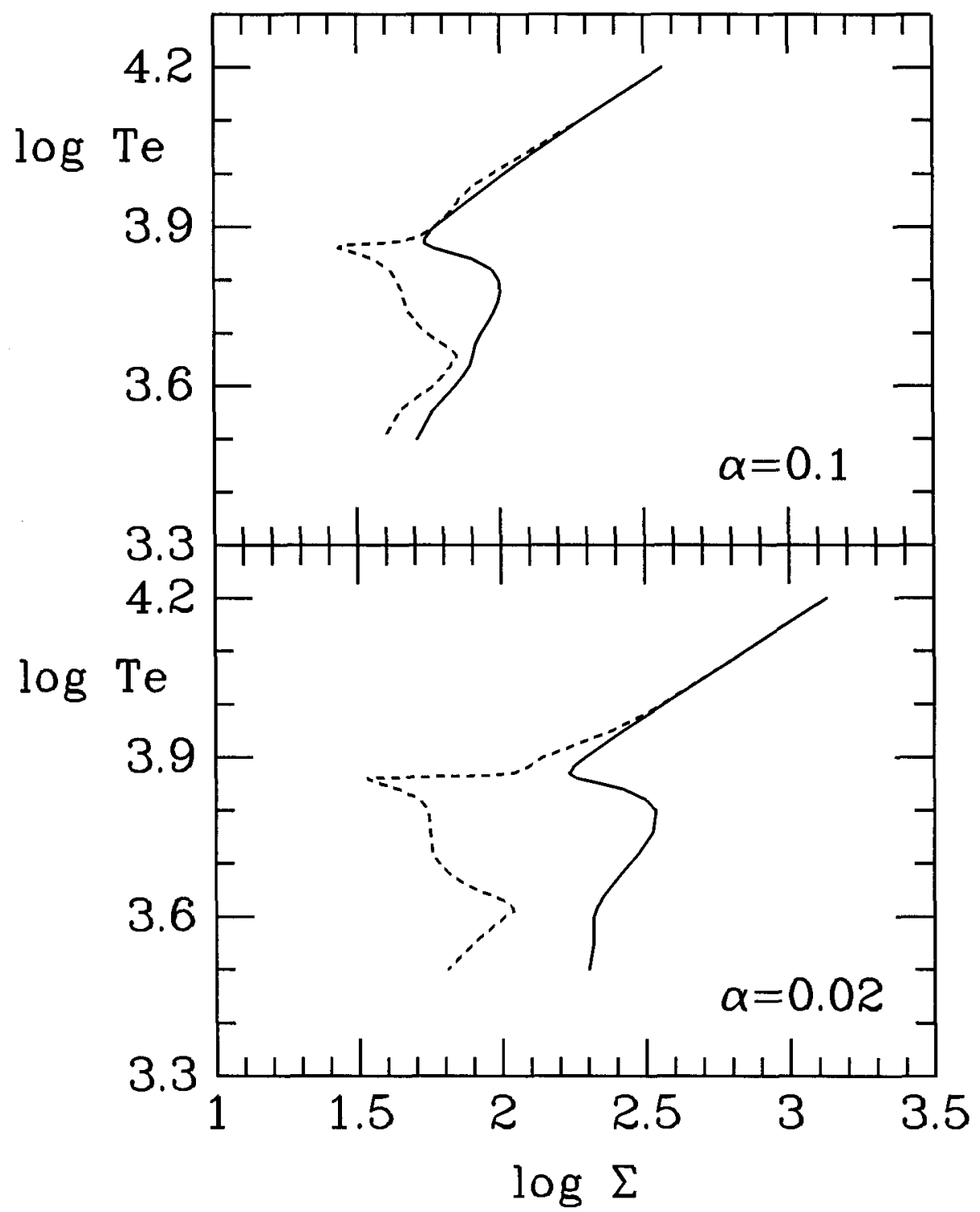

Figure 1. Examples of $\Sigma-T_{e}$ relations calculated with standard $\alpha$-disk viscosity (solid lines) and with standard $\alpha$-disk viscosity plus additional convective viscosity (solid lines). Model parameters are: $M=1 M_{\odot}$ and $R=1 \times 10^{10} \mathrm{~cm}$. 
the standard $\alpha$-disk case: instabilities in the disk occur in rapid succession, preventing their development into well defined outbursts separated by periods of quiescence.

\section{Discussion}

We begin by noticing that, in order to reproduce the dwarf nova behavior, the shape of the $\Sigma-T_{e}$ relation must be such as to make the disk instability recurrence time longer than the disk's viscous time scale. This can be accomplished only if the amount of mass that can be stored in the disk "on the cool branch" prior to the onset of an instability is large (note that this amount is roughly proportional to $\dot{M} \Delta t$, where $\dot{M}$ is the mass transfer rate and $\Delta t$ is the length of the outburst cycle). This implies that, compared to the standard $\alpha$-disk case with $\alpha_{\text {cool }}=\alpha_{h o t}$, two conditions must be fulfilled:

- $\Delta \log \Sigma=\log \Sigma_{m a x}-\log \Sigma_{m i n}$, must be larger, and

- $\Sigma_{\max }$ must also be larger.

Models including the convective viscosity meet only the first condition.

Figure 1 suggests that the second condition could be satisfied with very low values of $\alpha$, say $\alpha<0.01$. Within a single $\alpha$ approach, however, such low values are clearly unacceptable as leading to exceedingly long viscous time scales during outbursts. In fact, direct estimates of $\alpha$, based on the shapes of the outburst curves, give $\alpha_{\text {hot }} \approx 0.2$ (e.g. Smak 1984).

Acknowledgments. This work has been supported in part by a Grant 2 P03D 01111 (1996) from the Polish Committee for Scientific Research (KBN).

\section{References}

Cannizzo, J.K. \& Cameron, A.G.W. 1988, ApJ, 330, 327

Duschl, W.J. 1989, A\&A, 225, 105

Lin, D.N.C. \& Papaloizou, J. 1980, MNRAS, 191, 37

Osaki, Y. 1984, PASP, 108, 39

Paczyński, B. 1976, Comments Ap., 6, 95

Smak, J. 1982, Acta Astr., 32, 199

Smak, J. 1984, Acta Astr., 34, 161

Stone, J.M. \& Balbus, S.A. 1996, ApJ, 464, 364 